\title{
A stochastic-deterministic coupling method for continuum mechanics
}

\author{
R. Cottereau*, D. Clouteau, H. Ben Dhia, C. Zaccardi \\ Laboratoire MSSMat UMR 8579, École Centrale Paris, CNRS, France
}

\begin{abstract}
In this paper, we present a novel approach that allows to couple a deterministic continuum model with a stochastic continuum one. The coupling strategy is performed in the Arlequin framework, which is based on a volume coupling and a partition of the energy. A suitable functional space is chosen for the weak enforcement of the continuity between the two models. The choice of this space ensures that the mean of the stochastic solution equals the deterministic solution point-wise, and enforces appropriate boundary conditions on the stochastic dimension. The proof of the existence of the solution of the mixed problem is provided. The numerical strategy is also reviewed, in particular with a view at the Monte Carlo method. Finally, examples show the interest of the method, and possible strategies for use in adaptive modeling. Keywords: Multiscale method, Coupling method, Stochastic mechanics, Homogenization, Arlequin method
\end{abstract}

*regis.cottereau@ecp.fr 


\section{Introduction}

Classical deterministic models provide global predictions that are satisfactory for many industrial applications. However, when one is interested in a very localized behavior or quantity, or when multiscale phenomena come into play, these models may not be sufficient. For instance, the limited heterogeneity of a material modeled as a continuum might have no influence on its behavior on a large scale, while the study of a local stress intensity factor would strongly depend on the local heterogeneity of the mechanical parameters. Unfortunately, for these problems, the information necessary to parameterize the relevant, very complex, models is usually not available. Stochastic methods have therefore been proposed and now appear unavoidable in multiscale modeling.

Although the use of stochastic models and methods has expanded rapidly in the last decades, the related numerical costs are still often prohibitive. Hence, the application of these methods in a complex or industrial context remains limited. An important field of research is therefore concerned with the reduction of the costs associated with the use of stochastic methods, for example by using iterative methods specially adapted to the structure of the matrices arising in the Stochastic Finite Element (FE) method [1, 2], using reduced bases for the representation of random fields [3], or using special domain decomposition techniques for parallel resolution on clusters of computers [4].

The present paper proposes an alternative to these purely mathematical/numerical approaches through the coupling of two models: one deterministic and one stochastic. The general goal is that of modeling a global 
problem in a mean or homogeneous way where it yields sufficient accuracy, while retaining a stochastic model where needed. Hence, additional complexity is added in the model only where required, and the general approach is both more elegant and numerically cheaper than a global all-over stochastic model would be. Further, the cuts on computational costs mean that industrial applications come within reach.

The core idea for this paper, which is the choice of the operator and functional space for the coupling (Section 3.2) was proposed originally in [5]. It is here further described, in particular by adding the proof of existence and uniqueness of a solution for the mixed problem (Theorem 3.1), and showing how a Monte Carlo approach can be considered for the resolution of that problem (Section 3.4.2).

This work is closely related to two previous works in the literature $[6,7]$. However, in [6], the theoretical basis, which is different from the Arlequin formulation, is less general. In particular, it is only aimed at coupling a deterministic Boundary Element method with a stochastic FE method. In the recent work [7], the authors aim at coupling two stochastic models, one continuous, and one atomistic. However, many theoretical questions are left out. In particular, the coupling is performed between realizations of the stochastic operators, while we try to describe here the coupling at the level of the stochastic operators.

In the first part of this paper, we will present each of the two models that will be used: a deterministic continuum model with constant parameters (the "classical" one), and its stochastic counterpart, where the parameter varies randomly in space and is modeled as a random field. This first section will 
be concluded by a brief review of the uses and limitations of each of these models, taken separately, and the interest of using coupling approaches. The second part will describe the main novelty of the paper, that is the description of a general coupling approach for the two models described above, in the Arlequin framework $[14,15]$. Finally, applications in 1D and 2D will show the efficiency and interest of the method. In particular, a first hint at the use of this approach in the context of adaptivity will be described.

\section{Description of the two mono-models}

In this section, we describe the two models that will be considered in this paper: a continuum scalar mechanics model, with deterministic coefficients, and the same type of model with stochastic coefficients. Some indications will also be given concerning the uses and limitations of each of these two models in physical applications. The two models that are described in this section will be referred to as mono-models, in opposition to the coupled model that will be considered in the next section. In the section of applications, we will compare extensively the solutions obtained using the coupled model with those obtained with each of the two mono-models.

\subsection{The deterministic continuum mono-model}

Let us consider a domain $\Omega$ of $\mathbb{R}^{d}$, with outgoing normal vector $n$ and smooth boundary $\partial \Omega$, separated into Dirichlet and Neumann boundaries $\Gamma_{D}$ and $\Gamma_{N}$, such that $\Gamma_{D} \cup \Gamma_{N}=\partial \Omega, \Gamma_{D} \cap \Gamma_{N}=\emptyset$, and $\Gamma_{D} \neq \emptyset$ (figure 1, left). We consider Poisson's equation, with a deterministic scalar parameter $\underline{K}>0$, considered here constant, a bulk loading field $f(x)$, defined on $\Omega$, and a surface loading field $g_{n}(x)$, defined on $\Gamma_{N}$. Supposing for notational 

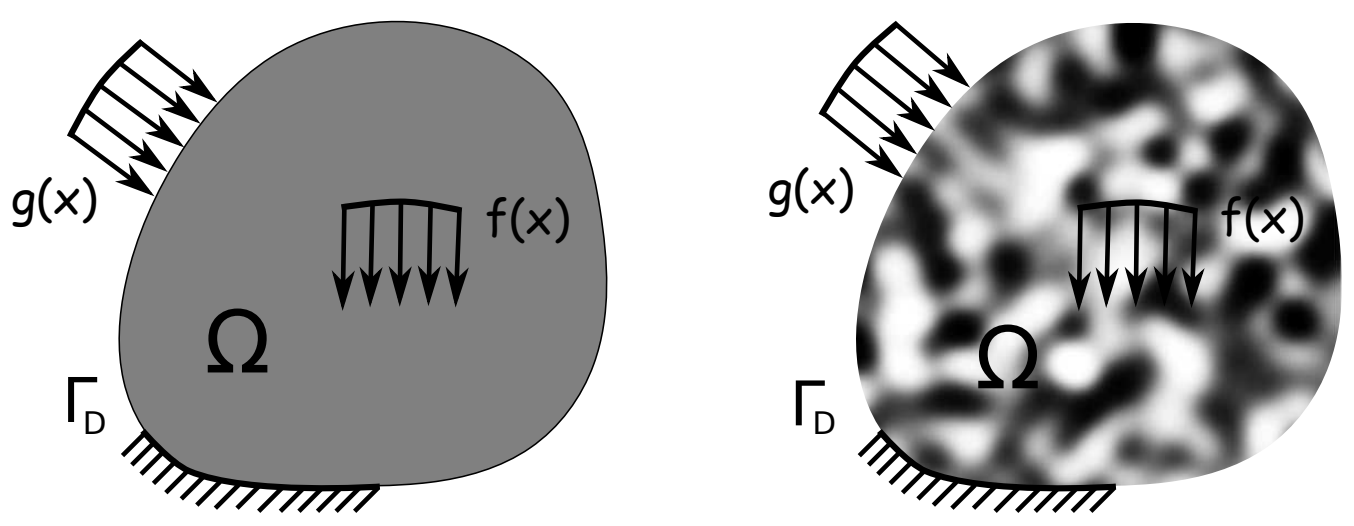

Figure 1: Description of the two mono-models: deterministic mono-model with constant coefficient $\underline{K}$ (left) and stochastic mono-model with heterogeneous coefficient $\boldsymbol{K}(x)$ (right)

simplicity that the Dirichlet boundary condition is homogeneous, the weak formulation for this problem reads: find $u \in \mathcal{V}$ such that

$$
a(u, v)=\ell(v), \forall v \in \mathcal{V},
$$

where $a: \mathcal{V} \times \mathcal{V} \rightarrow \mathbb{R}$ and $\ell: \mathcal{V} \rightarrow \mathbb{R}$ are defined, respectively, by $a(u, v)=$ $\int_{\Omega} \underline{K} \nabla u \cdot \nabla v d x$, and $\ell(v)=\int_{\Omega} f v d x+\int_{\Gamma_{N}} g_{n} v d x$, and

$$
\mathcal{V}=\left\{v \in \mathcal{H}^{1}(\Omega), v_{\mid \Gamma_{D}}=0\right\} .
$$

Endowed with the inner product $(u, v)_{\mathcal{V}}=\int_{\Omega} \nabla u \cdot \nabla v d x$, and associated norm $\|u\|_{\mathcal{V}}^{2}=\int_{\Omega}|\nabla u|^{2} d x, \mathcal{V}$ is a Hilbert space. The problem (1) can be shown to have a unique solution $u$, for instance using Lax-Milgram theorem [8, chapter 2]. This unique solution can be approximated, for example, by the Finite Element method.

\subsection{The stochastic continuum mono-model}

Let us now consider the same domain $\Omega$, but this time with a random fluctuating mechanical parameter. Let us model this parameter by a random 
field $\boldsymbol{K} \in \mathcal{L}^{2}\left(\Theta, L^{\infty}(\Omega)\right)$, where $(\Theta, \mathcal{F}, P)$ is a complete probability space, with $\Theta$ a set of outcomes, $\mathcal{F}$ a $\sigma$-algebra of events of $\Theta$, and $P: \mathcal{F} \rightarrow[0,1]$ a probability measure. We additionally assume (as in [9] for example) that this field is bounded and uniformly coercive, that is to say $\exists K_{\min }, K_{\max } \in$ $(0,+\infty)$, such that

$$
0<K_{\min } \leq \boldsymbol{K}(x) \leq K_{\max }<\infty, \forall x \in \Omega, \text { almost surely }
$$

The weak formulation of the corresponding stochastic boundary value problem reads: find $\boldsymbol{u} \in \mathcal{W}$ such that

$$
\mathcal{A}(\boldsymbol{u}, \boldsymbol{v})=\mathcal{L}(\boldsymbol{v}), \forall \boldsymbol{v} \in \mathcal{W}
$$

where $\mathcal{A}: \mathcal{W} \times \mathcal{W} \rightarrow \mathbb{R}$ and $\mathcal{L}: \mathcal{W} \rightarrow \mathbb{R}$ are defined, respectively, by $\mathcal{A}(\boldsymbol{u}, \boldsymbol{v})=\mathrm{E}\left[\int_{\Omega} \boldsymbol{K} \nabla \boldsymbol{u} \cdot \nabla \boldsymbol{v} d x\right]$, and $\mathcal{L}(\boldsymbol{v})=\int_{\Omega} f \mathrm{E}[\boldsymbol{v}] d x+\int_{\Gamma_{N}} g_{n} \mathrm{E}[\boldsymbol{v}] d x$, $\mathrm{E}[\cdot]=\int_{\Theta} \cdot d P$ denotes the mathematical expectation,

$$
\mathcal{W}=\mathcal{L}^{2}(\Theta, \mathcal{V})
$$

and $\mathcal{V}$ is defined in Eq. (2). Endowed with the inner product $(\boldsymbol{u}, \boldsymbol{v})_{\mathcal{W}}=$ $\mathrm{E}\left[(\boldsymbol{u}, \boldsymbol{v})_{\mathcal{V}}\right]=\mathrm{E}\left[\int_{\Omega} \nabla \boldsymbol{u} \cdot \nabla \boldsymbol{v} d x\right]$, and associated norm $\|\boldsymbol{u}\|_{\mathcal{W}}^{2}=\mathrm{E}\left[\|\boldsymbol{u}\|_{\mathcal{V}}^{2}\right]=$ $\mathrm{E}\left[\int_{\Omega}|\nabla \boldsymbol{u}|^{2} d x\right], \mathcal{W}$ is a Hilbert space.

As in the previous case, using Lax-Milgram theorem, it can be proved that this problem has a unique solution $\boldsymbol{u}$ (see for instance [9]). An approximation of that solution can then be obtained, for example, by using a Stochastic FE method [10, 11] or a Monte Carlo approach [12].

Remark 2.1. We assume here that the loads $f$ and $g$ are deterministic but this should not be seen as a restriction of the method. In particular, the 
mixed formulation of the next section and its numerical approximation can be developed with both the parameter $\boldsymbol{K}(x)$ and the loads modeled as random fields.

Remark 2.2. The existence and uniqueness of the solution of the above stochastic boundary value problem can also be proved with less constraining boundary conditions. In particular, the case when

$$
\mathcal{W}=\left\{\mathcal{L}^{2}\left(\Theta, \mathcal{H}^{1}(\Omega)\right) ; \mathrm{E}[\boldsymbol{v}]=0, \forall x \in \Gamma_{D} ; \int_{\Gamma_{D}} \boldsymbol{v} d x=0, a . s .\right\}
$$

still works. The homogeneous boundary condition is therefore not imposed anymore almost surely and almost everywhere. Rather, the space average of the displacement over the Dirichlet boundary cancels almost surely. This type of boundary condition is similar to what is done within the Arlequin framework in section 3.2.

\subsection{Use of the mono-models and interest of coupling approaches}

The mono-model described in section 2.1 is interesting when the material is considered on a scale at which homogenization can take place. This statement is intrinsically linked to the quantities of interest that we aim to evaluate. In particular, the estimation of the average displacement over a given area might be well evaluated using such a homogenized mono-model. On the other hand, considering local quantities with this mono-model is not adequate. For example, following the path of the tip of a fracture can probably not be performed using this model. As a general pattern, the deterministic mono-model of section 2.1 will be appropriate for the evaluation of average quantities in macro-scale problems. In that setting, the FE method 
can be very efficiently implemented and yields accurate results for a relatively low cost.

The stochastic mono-model tries to take into account, to some extent, the inherent heterogeneity of the material, without falling into the pits of

- really modeling the material at a smaller scale, by considering a fully different physical setting, e.g. polycrystalline mechanics;

- having to identify the exact value of the parameter field in each point in space, which, in a fully heterogeneous setting, would result in a infinite number of scalar parameters to be identified.

By introducing a stochastic field for the parameter, we consider a random heterogeneous field that is parameterized only by a few quantities (typically, the mean, the autocorrelation, and the correlation length) and takes into account a possible uncertainty related to the identification of the parameter field. This approach is much richer than the previous homogeneous one, but at the cost of a more expensive solution process. Roughly speaking, we have added one dimension to the size of the problem.

Often, the solution of the refined model is too expensive, while the details provided by the coarse model are not sufficient. Hence, we propose in the next section a coupled approach, for which both models are considered, each on a different (and overlapping) part of the total domain. Note that this requires that each of the models be appropriate where it expresses itself. In particular, this means that only local features of the refined model are required for a good evaluation of the global solution (see the applications in section 4 for more details). 
Remark 2.3. The Arlequin method was originally designed to perform the coupling of different physical models (e.g. shell and 3D continuum [13], continuum and atomistic [7], ...). Hence the generalization of the approach presented in this paper for continuum-continuum coupling to different types of physical models, for which one of the two is stochastic, is not expected to yield any additional difficulty. Other examples of coupling will be presented in forthcoming papers.

\section{A general deterministic-stochastic coupling approach}

In this section, we describe the construction of the coupled model, including features of the deterministic continuum mono-model on part of the domain, and features of the stochastic continuum mono-model on another one. As this construction is performed in the Arlequin framework $[14,15,13,16]$, we first recall this method on a more classical deterministic-deterministic continuum model. Then, the stochastic-deterministic case, which is the core of this paper, is described.

\subsection{The Arlequin method for coupling two deterministic continuum models}

Let us introduce two overlapping subdomains of the domain $\Omega$ : $\Omega_{1}$, on which the first model will express itself, and $\Omega_{2}$, for the second model. We further select a subdomain $\Omega_{c}$ of the overlap $\Omega_{1} \cap \Omega_{2}$ over which the two models will communicate. The parts over which only one model is described and the part of the overlap over which no communication takes place $\left(\Omega_{1} \cup\right.$ $\left.\Omega_{2}\right) \backslash \Omega_{c}$ will be called free, in the sense that each model expresses itself with no constraint there. 


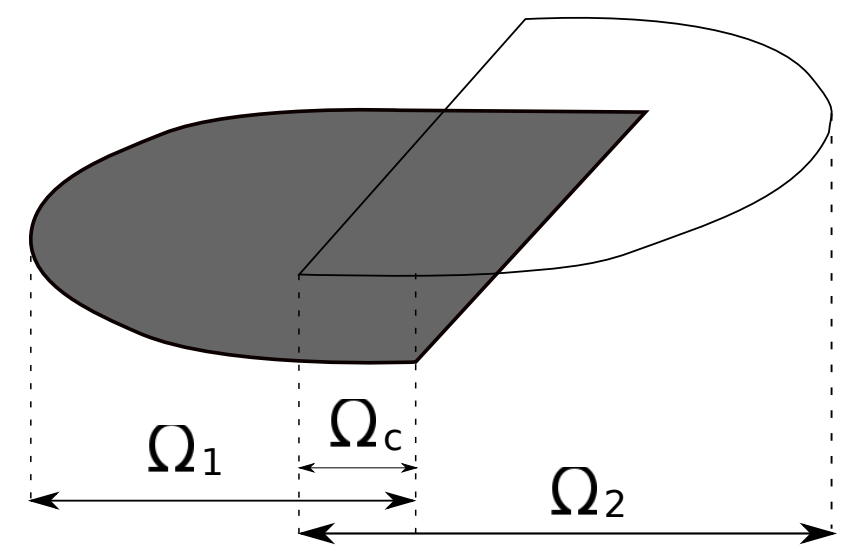

Figure 2: Definition of the different subdomains, with emphasis on the coupling area $\Omega_{c}$ on which both models express themselves.

We consider here the case where the two models are deterministic and similar. This is useful in the context of mesh refinement, as the two models are the same but are not discretized similarly $[14,15,13]$. Hence, a coarse finite element basis (supported by a coarse mesh) will be used on $\Omega_{1}$ to account for large scale deformations and stresses, and a refined one (supported on a fine mesh) will be used on $\Omega_{2}$ to reproduce more accurately some local effects of interest.

The Arlequin method allows to couple these two models through the resolution of the following mixed problem: find $\left(u_{1}, u_{2}, \Phi\right) \in \mathcal{V}_{1} \times \mathcal{V}_{2} \times \mathcal{V}_{c}$ such that

$$
\begin{cases}a_{1}\left(u_{1}, v\right)+C(\Phi, v)=\ell_{1}(v), & \forall v \in \mathcal{V}_{1} \\ a_{2}\left(u_{2}, v\right)-C(\Phi, v)=\ell_{2}(v), & \forall v \in \mathcal{V}_{2} \\ C\left(\Psi, u_{1}-u_{2}\right)=0, & \forall \Psi \in \mathcal{V}_{c}\end{cases}
$$

where the bilinear forms $a_{1}: \mathcal{V}_{1} \times \mathcal{V}_{1} \rightarrow \mathbb{R}, a_{2}: \mathcal{V}_{2} \times \mathcal{V}_{2} \rightarrow \mathbb{R}$ and $C$ : $\mathcal{V}_{c} \times \mathcal{V}_{c} \rightarrow \mathbb{R}$ are defined, respectively, by $a_{1}(u, v)=\int_{\Omega} \alpha_{1} \underline{K} \nabla u \cdot \nabla v d x$, 
$a_{2}(u, v)=\int_{\Omega_{2}} \alpha_{2} \underline{K} \nabla u \cdot \nabla v d x$, and

$$
C(u, v)=\int_{\Omega_{c}}\left(\kappa_{0} u v+\kappa_{1} \nabla u \cdot \nabla v\right) d x
$$

with $\kappa_{0}$ and $\kappa_{1}$ two constants (see for example [13] for details), the linear forms $\ell_{1}: \mathcal{V}_{1} \rightarrow \mathbb{R}$ and $\ell_{2}: \mathcal{V}_{2} \rightarrow \mathbb{R}$ and defined, respectively, by $\ell_{1}(v)=$ $\int_{\Omega_{1}} \alpha_{1} f v d x+\int_{\Gamma_{N}} \alpha_{1} g_{n} v d x$ and $\ell_{2}(v)=\int_{\Omega_{2}} \alpha_{2} f v d x+\int_{\Gamma_{N}} \alpha_{2} g_{n} v d x$, the weights are chosen such that (see figure 3 )

$$
\left\{\begin{array}{ll}
\alpha_{1}, \alpha_{2} \geq 0 & \text { in } \Omega_{1} \cup \Omega_{2} \\
\alpha_{1}+\alpha_{2}=1 & \text { in } \Omega_{1} \cup \Omega_{2} \\
\alpha_{1}, \alpha_{2} \text { constant } & \text { in }\left(\Omega_{1} \cup \Omega_{2}\right) \backslash \Omega_{c}
\end{array},\right.
$$

and the functional spaces are $\mathcal{V}_{1}=\left\{v \in \mathcal{H}^{1}\left(\Omega_{1}\right), v_{\mid \Gamma_{D}}=0\right\}, \mathcal{V}_{2}=\{v \in$ $\left.\mathcal{H}^{1}\left(\Omega_{2}\right)\right\}$ and $\mathcal{V}_{c}=\left\{v \in \mathcal{H}^{1}\left(\Omega_{c}\right)\right\}$.

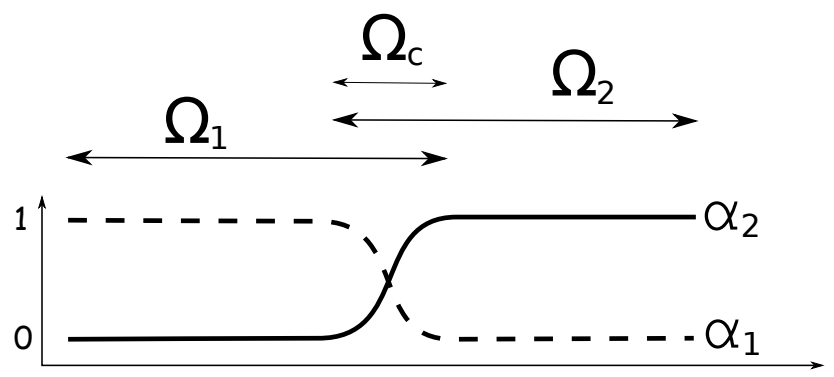

Figure 3: Example of definition of the functions $\alpha_{1}$ and $\alpha_{2}$ in the case when $\Omega_{2}$ is not embedded in $\Omega_{1}$, as a function of the position, in $1 \mathrm{D}$.

With reasonable assumptions (in particular, that $\Omega_{c} \neq \emptyset$ ), the Arlequin system (7) can be shown to have a unique solution [15]. This solution can be approximated by the Finite Element method. 
Remark 3.1. For notational simplicity, this has been derived in the case where the patch $\Omega_{2}$ is totally included inside the domain $\Omega$. In particular, the patch does not intersect the Dirichlet boundary condition, i.e. $\partial \Omega_{2} \cap \Gamma_{D}=\emptyset$. More general results can be obtained [16].

\subsection{The Arlequin method for deterministic-stochastic coupling}

We now wish to superpose, in the Arlequin framework, the following two models: a deterministic continuum model (as in Sec. 2.1), in $\Omega_{1}$; and a stochastic continuum model (as in Sec. 2.2), in $\Omega_{2}$. We will therefore consider two models of the parameter field: a deterministic one, $\underline{K}$, assumed (for simplicity) constant on the domain $\Omega_{1}$; and a stochastic one, $\boldsymbol{K}(x)$, modeled as a random field on $\Omega_{2}$. We further assume that $\boldsymbol{K}$ verifies on $\Omega_{2}$ conditions similar to the ones described in Sec. 2.2 on $\Omega$. Finally, for the coupling approach to make sense, the two models should correspond one to the other, through homogenization [18]. In particular, in 1D, one should have $\underline{K}=\mathrm{E}\left[\boldsymbol{K}^{-1}\right]^{-1}$.

The stochastic-deterministic Arlequin problem reads: find $\left(u_{1}, \boldsymbol{u}_{2}, \boldsymbol{\Phi}\right) \in$ $\mathcal{V}_{1} \times \mathcal{W}_{2} \times \mathcal{W}_{c}$ such that

$$
\begin{cases}a_{1}\left(u_{1}, v\right)+C(\boldsymbol{\Phi}, v)=\ell_{1}(v), & \forall v \in \mathcal{V}_{1} \\ \mathcal{A}_{2}\left(\boldsymbol{u}_{2}, \boldsymbol{v}\right)-C(\boldsymbol{\Phi}, \boldsymbol{v})=\mathcal{L}_{2}(\boldsymbol{v}), & \forall \boldsymbol{v} \in \mathcal{W}_{2} \\ C\left(\boldsymbol{\Psi}, u_{1}-\boldsymbol{u}_{2}\right)=0, & \forall \boldsymbol{\Psi} \in \mathcal{W}_{c}\end{cases}
$$

where the bilinear forms $a_{1}: \mathcal{V}_{1} \times \mathcal{V}_{1} \rightarrow \mathbb{R}, \mathcal{A}_{2}: \mathcal{W}_{2} \times \mathcal{W}_{2} \rightarrow \mathbb{R}$, and $C: \mathcal{W}_{c} \times \mathcal{W}_{c} \rightarrow \mathbb{R}$ are defined by

$$
a_{1}(u, v)=\int_{\Omega_{1}} \alpha_{1} \underline{K} \nabla u \cdot \nabla v d x,
$$




$$
\mathcal{A}_{2}(\boldsymbol{u}, \boldsymbol{v})=\mathrm{E}\left[\int_{\Omega_{2}} \alpha_{2} \boldsymbol{K} \nabla \boldsymbol{u} \cdot \nabla \boldsymbol{v} d x\right]
$$

and

$$
C(\boldsymbol{u}, \boldsymbol{v})=\mathrm{E}\left[\int_{\Omega_{c}}\left(\kappa_{0} \boldsymbol{u v}+\kappa_{1} \nabla \boldsymbol{u} \cdot \nabla \boldsymbol{v}\right) d x\right],
$$

where the linear forms $\ell_{1}: \mathcal{V}_{1} \rightarrow \mathbb{R}$ and $\mathcal{L}_{2}: \mathcal{W}_{2} \rightarrow \mathbb{R}$ are defined, respectively, by $\ell_{1}(v)=\int_{\Omega_{1}} \alpha_{1} f v d x+\int_{\Gamma_{N}} \alpha_{1} g_{n} v d x$ and $\mathcal{L}_{2}(\boldsymbol{v})=\int_{\Omega_{2}} \alpha_{2} f \mathrm{E}[\boldsymbol{v}] d x+$ $\int_{\Gamma_{N}} \alpha_{2} g_{n} \mathrm{E}[\boldsymbol{v}] d x$, and the functional spaces $\mathcal{W}_{2}$ and $\mathcal{W}_{c}$ are given by

$$
\mathcal{W}_{2}=\mathcal{L}^{2}\left(\Theta, \mathcal{V}_{2}\right)
$$

and

$$
\mathcal{W}_{c}=\left\{\psi(x)+\boldsymbol{\theta} \mathbb{I}_{c}(x) \mid \psi \in \mathcal{V}_{c}, \int_{\Omega_{c}} \psi d x=0, \boldsymbol{\theta} \in \mathcal{L}^{2}(\Theta, \mathbb{R})\right\},
$$

with $\mathcal{V}_{2}=\mathcal{H}^{1}\left(\Omega_{2}\right)$ and $\mathcal{V}_{c}=\mathcal{H}^{1}\left(\Omega_{c}\right)$, and where the indicator function $\mathbb{I}(x)$ is such that $\mathbb{I}_{c}\left(x \in \Omega_{c}\right)=1$ and $\mathbb{I}_{c}\left(x \notin \Omega_{c}\right)=0$. The condition imposed here on $\psi$ is not necessary for the definition of the space $\mathcal{W}_{c}$. It is however presented here in view of the redundancy that arises when the discretization of the corresponding fields is contemplated. Indeed, a deterministic rigid body mode (here, a constant field) can be described equivalently as $(\psi=$ $0, \boldsymbol{\theta}=1$ ), where $\boldsymbol{\theta}=1$ is taken as dirac function in the stochastic dimension, or $(\psi=\mathbb{I}(x), \boldsymbol{\theta}=0)$. One could instead use the condition $\mathrm{E}[\boldsymbol{\theta}]=0$, but this implies a global condition in the stochastic dimension, and is hence not appropriate for the Monte Carlo solution proposed in section 3.4.2.

Note that the space $\mathcal{W}_{c}$ can be seen as composed of random fields with a spatially varying mean and perfectly spatially correlated randomness. Thanks to the specific structure of that space, the last line of the system (10) can be 
written equivalently, $\forall \boldsymbol{\Psi}=\psi(x)+\boldsymbol{\theta} \mathbb{I}_{c}(x) \in \mathcal{W}_{c}$,

$$
\begin{aligned}
0 & =C\left(\boldsymbol{\Psi}, u_{1}-\boldsymbol{u}_{2}\right) \\
& =\mathrm{E}\left[\int_{\Omega_{c}}\left(\kappa_{0}\left(\psi+\boldsymbol{\theta} \mathbb{I}_{c}\right)\left(u_{1}-\boldsymbol{u}_{2}\right)+\kappa_{1} \nabla \psi \cdot \nabla\left(u_{1}-\boldsymbol{u}_{2}\right)\right) d x\right] \\
& =C\left(\mathrm{E}[\boldsymbol{\Psi}], u_{1}-\mathrm{E}\left[\boldsymbol{u}_{2}\right]\right)+\kappa_{0} \mathrm{E}\left[\boldsymbol{\theta} \int_{\Omega_{c}}\left(u_{1}-\boldsymbol{u}_{2}\right) d x\right] .
\end{aligned}
$$

Therefore, this condition imposes that the (ensemble) average of the field $\boldsymbol{u}_{2}$ should be equal to the field $u_{1}$, in all points of $\Omega_{c}$, and that the variability of the space average quantity $\int_{\Omega_{c}}\left(\mathrm{E}\left[\boldsymbol{u}_{2}\right]-\boldsymbol{u}_{2}\right) d x$ should cancel. In other words, this means that some degree of homogenization takes place within the coupling zone. In particular, if that zone is not big enough with respect to the correlation lengths of the fields $\boldsymbol{K}(x)$ or $\boldsymbol{u}_{2}(x)$, the Arlequin scheme is expected to yield results that would be different from those obtained with the stochastic monomodel. It would mean that there is not enough localization of the variability and stochasticity for the Arlequin scheme to make sense.

Remark 3.2. The indicator function $\mathbb{I}(x)$ is a generator of the kernel of the acoustic operator we are considering here. If we were to consider elasticity, the definition of the mediator space would be the superposition of a spacefluctuating average (like here), and six rigid-body movements with random coefficients.

Theorem 3.1. If the weight functions do not cancel on their respective domains of definition, including the overlapping area (i.e. $\exists \alpha_{0}>0$ such that $\alpha_{0}<\alpha_{1}(x)$ in $\Omega_{1}$ and $\alpha_{0}<\alpha_{2}(x)$ in $\left.\Omega_{2}\right)$, the problem (10) admits a unique solution.

Proof. To prove the existence and uniqueness of this system of equations, we 
will follow the theory of Brezzi [19], and more specifically the steps of [14, 15], where the reader can find more details on a similar proof. To come closer to the format used in the cited papers, we will rewrite the system as: find $(\boldsymbol{u}, \Phi) \in \mathcal{V} \times \mathcal{W}_{c}$ such that

$$
\left\{\begin{array}{ll}
\mathcal{A}(\boldsymbol{u}, \boldsymbol{v})+\hat{C}(\boldsymbol{\Phi}, \boldsymbol{v})=\mathcal{L}(\boldsymbol{v}), & \forall \boldsymbol{v} \in \mathcal{V} \\
\hat{C}(\boldsymbol{\Psi}, \boldsymbol{u})=0, & \forall \boldsymbol{\Psi} \in \mathcal{W}_{c}
\end{array},\right.
$$

with $\mathcal{V}=\mathcal{V}_{1} \times \mathcal{W}_{2}, \mathcal{A}(\boldsymbol{u}, \boldsymbol{v})=a_{1}\left(u_{1}, v_{1}\right)+\mathcal{A}_{2}\left(\boldsymbol{u}_{2}, \boldsymbol{v}_{2}\right)$, with $\boldsymbol{u}=\left(u_{1}, \boldsymbol{u}_{2}\right) \in \mathcal{V}$ and $\boldsymbol{v}=\left(v_{1}, \boldsymbol{v}_{2}\right) \in \mathcal{V}, \hat{C}(\boldsymbol{\Phi}, \boldsymbol{u})=C\left(\boldsymbol{\Phi}, u_{1}-\boldsymbol{u}_{2}\right)$, and $\mathcal{L}(\boldsymbol{v})=\ell_{1}\left(v_{1}\right)+\mathcal{L}_{2}\left(\boldsymbol{v}_{2}\right)$. To ensure the existence and uniqueness of a solution to this problem, we must check a property of coercivity of the bilinear form $\mathcal{A}$ on the kernel of $\hat{C}$ (with respect to the second argument), and the inf-sup condition on $\hat{C}$ (the continuity of the linear and bilinear forms defined here is trivial).

To check the coercivity of the bilinear form, we construct the sequence of normalized functions $\boldsymbol{v}^{n}=\left(v_{1}^{n}, \boldsymbol{v}_{2}^{n}\right)$ in the kernel $N_{\mathcal{W}_{c}}(\hat{C})=\{\boldsymbol{v} \in \mathcal{V} \mid \forall \boldsymbol{\Psi} \in$ $\left.\mathcal{W}_{c}, \hat{C}(\Psi, \boldsymbol{v})=0\right\}$ (with $\left\|\boldsymbol{v}^{n}\right\|=1, \forall n>0$ ) that converges strongly to $\underline{\boldsymbol{v}}=\left(v_{1}, \boldsymbol{v}_{2}\right)$, with $\nabla v_{1}=0$ almost everywhere on $\Omega_{1}$ and $\nabla \boldsymbol{v}_{2}=0$ almost everywhere in $\Omega_{2}$ and almost surely. The former condition, along with the homogeneous Dirichlet boundary condition in $\mathcal{V}_{1}$ ensures that $v_{1}=0$ almost everywhere on $\Omega_{1}$. The latter condition implies, by definition of $\mathcal{W}_{c}$ (see Eq. (15)) and a continuity argument, that $\boldsymbol{v}_{2} \in \mathcal{W}_{c}$. Because $v_{1}=0$, and because the rigid body modes for $\boldsymbol{u}_{2}$ on $\Omega_{c}$ are contained in $\mathcal{W}_{c}$, this implies $\hat{C}\left(\boldsymbol{u}_{2}, \boldsymbol{u}\right)=-C\left(\boldsymbol{u}_{2}, \boldsymbol{u}_{2}\right)=0$, and, since $C$ is a scalar product, $\boldsymbol{u}_{2}=0$ almost everywhere in $\Omega_{c}$ and almost surely. The extension to $\Omega_{2}$ comes again from $\nabla \boldsymbol{v}_{2}=0$. Hence, the limiting sequence verifies $\boldsymbol{v}=0$, which is in contradiction with the initial hypotheses, and proves the coercivity of $\mathcal{A}$ on 
$N_{\mathcal{W}_{c}}(\hat{C})$. The key concepts for this part of the proof are the positivity of $\alpha_{1}$ and $\alpha_{2}$ on their respective domains of definition, and the fact that the rigid body modes for $\boldsymbol{u}_{2}$ on $\Omega_{c}$ are contained in $\mathcal{W}_{c}$.

To prove the inf-sup condition on $\hat{C}$, we recall that, because $\mathcal{W}_{c}$ is included inside the restriction of $\mathcal{W}_{2}$ to the space support $\Omega_{c}$, it is possible to prolongate continuously any element $\boldsymbol{\Psi} \in \mathcal{W}_{c}$ into an element $\overline{\boldsymbol{\Psi}}_{2} \in \mathcal{W}_{2}$. Then, choosing $\boldsymbol{v}=\left(0,-\overline{\boldsymbol{\Psi}}_{2}\right) \in \mathcal{V}$, we have

$$
\sup _{\boldsymbol{v} \in \mathcal{V},\|\boldsymbol{v}\|_{\mathcal{V} \neq 0}} \frac{\hat{C}(\boldsymbol{\Psi}, \boldsymbol{v})}{\|\boldsymbol{v}\|_{\mathcal{V}}} \geq \frac{C\left(\boldsymbol{\Psi}, \overline{\boldsymbol{\Psi}}_{2}\right)}{\left\|\overline{\boldsymbol{\Psi}}_{2}\right\|_{\mathcal{W}_{2}}}
$$

where $\|\boldsymbol{v}\|_{\mathcal{V}}^{2}=\left\|v_{1}\right\|_{\mathcal{V}_{1}}^{2}+\left\|\boldsymbol{v}_{2}\right\|_{\mathcal{W}_{2}}^{2},\left\|v_{1}\right\|_{\mathcal{V}_{1}}^{2}=\int_{\Omega_{1}} v_{1}^{2}+\left|\nabla u_{1}\right|^{2} d x$, and $\left\|\boldsymbol{v}_{2}\right\|_{\mathcal{W}_{2}}^{2}=$ $\mathrm{E}\left[\int_{\Omega_{2}} \boldsymbol{v}_{2}^{2}+\left|\nabla \boldsymbol{v}_{2}\right|^{2} d x\right]$. Using the continuity of the prolongation operator, $\exists \gamma_{1}>0,\left\|\Psi_{2}\right\|_{\mathcal{W}_{2}} \leq \gamma_{1}\|\boldsymbol{\Psi}\|_{\mathcal{W}_{c}}$, where $\|\boldsymbol{\Psi}\|_{\mathcal{W}_{c}}^{2}=\mathrm{E}\left[\int_{\Omega_{c}} \boldsymbol{\Psi}_{2}^{2}+\left|\nabla \boldsymbol{\Psi}_{2}\right|^{2} d x\right]$. Further, the definition of $C$ (Eq. (13)) gives $C\left(\boldsymbol{\Psi}, \boldsymbol{\Psi}_{2}\right) \geq \min \left(\kappa_{0}^{2}, \kappa_{1}^{2}\right)\|\boldsymbol{\Psi}\|_{\mathcal{W}_{c}}^{2}$, which yields the desired inf-sup condition, with a positive constant $\gamma$ :

$$
\sup _{\boldsymbol{v} \in \mathcal{V},\|\boldsymbol{v}\|_{\mathcal{V}} \neq 0} \frac{\hat{C}(\boldsymbol{\Psi}, \boldsymbol{v})}{\|\boldsymbol{v}\|_{\mathcal{V}}} \geq \gamma\|\boldsymbol{\Psi}\|_{\mathcal{W}_{c}}
$$

Remark 3.3. At the beginning of this section, we introduced a condition of compatibility between the deterministic and stochastic models $\underline{K}=E\left[\boldsymbol{K}^{-1}\right]^{-1}$ (in 1D). However, this condition is not necessary for the mixed problem (10) to be well-posed. It is only necessary for the results to be meaningful, in the sense that the two models represent the same physical behavior at different scales. More precisely, in the classical Arlequin formulation, the weight functions $\alpha_{1}$ and $\alpha_{2}$ are requested to be a partition of unity over the coupling domain, while this is not the case anymore here. What is required is that the 
homogenization, at any point $x$ in the coupling zone, of $\alpha_{1} \underline{K}+\alpha_{2} \boldsymbol{K}$ be $\underline{K}$. In $1 D$ in particular, this means $\underline{K}^{-1}=E\left[\left(\alpha_{1}(x) \underline{K}+\alpha_{2}(x) \boldsymbol{K}(x)\right)^{-1}\right]$. More details on this and the convergence of the solution of the Arlequin problem to the solutions of the deterministic and stochastic mono-models will be given in a forthcoming paper.

\subsection{Resolution of the deterministic-stochastic coupled system}

In the previous section we presented the mixed problem (10) and proved that it admits a unique solution. Here, we describe two concurrent methods to compute this solution: one based on a finite element discretization of both space and random dimensions over an appropriate tensor functional basis, and one based on a classical finite element discretization for space, while the random dimension is treated through a Monte-Carlo approach. As the discretization in space is treated in the same manner for both approaches, we begin with this.

\subsection{Finite Element discretization in space}

We therefore associate to the domain $\Omega_{1}$ a mesh $\mathcal{T}_{1}$, composed of elements $E_{1}$, to the domain $\Omega_{2}$ a mesh $\mathcal{T}_{2}$, composed of elements $E_{2}$, and to the domain $\Omega_{c}$ a mesh $\mathcal{T}_{c}$, composed of elements $E_{c}$. We look for approximate functions of the elements of $\mathcal{V}_{1}, \mathcal{V}_{2}$ and $\mathcal{V}_{c}$ in the functional spaces

$$
\begin{gathered}
\mathcal{V}_{1}^{H}=\left\{v \in P_{1}\left(E_{1}\right), v_{\mid \Gamma_{D}}=0\right\}, \\
\mathcal{V}_{2}^{H}=\left\{v \in P_{1}\left(E_{2}\right)\right\},
\end{gathered}
$$

and

$$
\mathcal{V}_{c}^{H}=\left\{v \in P_{1}\left(E_{c}\right)\right\}
$$


composed of linear functions on each of the elements of the meshes. We then choose the bases $\left\{\mathrm{v}_{\ell}^{1}(\boldsymbol{x})\right\}_{1 \leq \ell \leq m_{1}},\left\{\mathrm{v}_{\ell}^{2}(\boldsymbol{x})\right\}_{1 \leq \ell \leq m_{2}}$, and $\left\{\mathrm{v}_{\ell}^{c}(\boldsymbol{x})\right\}_{1 \leq \ell \leq m_{c}}$ for the functions in $\mathcal{V}_{1}^{H}, \mathcal{V}_{2}^{H}$, and $\mathcal{V}_{c}^{H}$, respectively. We then introduce the matrices $\mathrm{A}_{1}, \mathrm{~A}_{2}, \mathrm{C}_{1}$, and $\mathrm{C}_{2}$, with elements

$$
\begin{gathered}
\mathrm{A}_{1, i j}=\int_{\Omega_{1}} \alpha_{1} \underline{K} \nabla \mathrm{v}_{i}^{1} \cdot \nabla \mathrm{v}_{j}^{1} d x \\
\mathbf{A}_{2, i j}=\int_{\Omega_{2}} \alpha_{2} \boldsymbol{K} \nabla \mathrm{v}_{i}^{2} \cdot \nabla \mathrm{v}_{j}^{2} d x \\
\mathrm{C}_{1, i j}=\int_{\Omega_{c}}\left(\kappa_{0} \mathrm{v}_{i}^{1} \mathrm{v}_{j}^{c}+\kappa_{1} \nabla \mathrm{v}_{i}^{1} \cdot \nabla \mathrm{v}_{j}^{c}\right) d x, \\
\mathrm{C}_{2, i j}=\int_{\Omega_{c}}\left(\kappa_{0} \mathrm{v}_{i}^{2} \mathrm{v}_{j}^{c}+\kappa_{1} \nabla \mathrm{v}_{i}^{2} \cdot \nabla \mathrm{v}_{j}^{c}\right) d x,
\end{gathered}
$$

and the vectors $\mathrm{S}_{1}, \mathrm{~S}_{2}$, and $\mathrm{S}_{c}$, with elements

$$
\begin{aligned}
\mathrm{S}_{1, i} & =\int_{\Omega_{c}} \mathrm{v}_{i}^{1} d x, \\
\mathrm{~S}_{2, i} & =\int_{\Omega_{c}} \mathrm{v}_{i}^{2} d x, \\
\mathrm{~S}_{c, i} & =\int_{\Omega_{c}} \mathrm{v}_{i}^{c} d x .
\end{aligned}
$$

The mixed system (10) can now be written, after space discretization only:

$$
\mathrm{E}\left[\left[\begin{array}{ccccc}
\mathrm{A}_{1} & 0 & \mathrm{C}_{1} & \kappa_{0} \mathrm{~S}_{1} & 0 \\
0 & \mathbf{A}_{2} & -\mathrm{C}_{2} & -\kappa_{0} \mathrm{~S}_{2} & 0 \\
\mathrm{C}_{1}^{T} & -\mathrm{C}_{2}^{T} & 0 & 0 & \mathrm{~S}_{c}^{T} \\
\kappa_{0} \mathrm{~S}_{1}^{T} & -\kappa_{0} \mathrm{~S}_{2}^{T} & 0 & 0 & 0 \\
0 & 0 & \mathrm{~S}_{c} & 0 & 0
\end{array}\right]\left[\begin{array}{c}
\mathrm{U}_{1} \\
\mathrm{U}_{2} \\
\Psi \\
\boldsymbol{\theta} \\
\Lambda
\end{array}\right]\right]=\left[\begin{array}{c}
\mathrm{F}_{1} \\
\mathrm{~F}_{2} \\
0 \\
0 \\
0
\end{array}\right]
$$

where the coordinates of the vectors $\mathrm{F}_{1}$ and $\mathrm{F}_{2}$ are given by $\mathrm{F}_{1, i}=\ell_{1}\left(\mathrm{v}_{i}^{1}\right)$ and $\mathrm{F}_{2, i}=\mathcal{L}_{2}\left(\mathrm{v}_{i}^{2}\right)$ and $\mathrm{U}_{1}, \mathrm{U}_{2}$ and $\Psi$ are the vectors of coordinates of $u_{1}, \boldsymbol{u}_{2}$, and 
the deterministic part of $\Phi$, in the bases of $\mathcal{V}_{1}^{H}, \mathcal{V}_{2}^{H}$, and $\mathcal{V}_{c}^{H}$, respectively. The vector $\Lambda$ contains the lagrange multipliers used to enforce the condition in the definition of $\mathcal{W}_{c}(15)$. We emphasize here that only $\mathbf{A}_{2}, \mathbf{U}_{2}$ and $\boldsymbol{\theta}$ are random.

\subsubsection{Spectral-like approaches}

We now discretize the random dimension using some spectral form, such as a polynomial chaos expansion [10]. In particular, we choose an approximating space as the span of the polynomial chaos basis of order $n$ and degree $p,\left\{\hat{\Gamma}_{\ell}[\boldsymbol{\xi}]\right\}_{1 \leq \ell \leq N}$, in conjunction with the previous bases for the space dimension. $N$ is the number of elements in the polynomial chaos basis, which depends both on $n$ and $p, \boldsymbol{\xi}$ is a vector of independent random variables not necessarily gaussian (see [20]), and the $\hat{\Gamma}_{\ell}$ are the corresponding orthogonal polynomials [20]. We expand the parameter field $\boldsymbol{K}$, the solution $\boldsymbol{u}_{2}$ and $\boldsymbol{\theta}$ in these bases, and finally obtain the matrix $\mathrm{A}_{2}$ for the stochastic part of the mixed system

$$
\mathbb{A}_{j \ell, J L}=\sum_{i=1}^{N} c_{i j J} \int_{\Omega_{2}} \alpha_{2}(x) k_{i}(x) \nabla \mathrm{v}_{\ell}^{2}(x) \cdot \nabla \mathrm{v}_{L}^{2}(x) d x
$$

where $c_{i j J}=\mathrm{E}\left[\hat{\Gamma}_{i}[\boldsymbol{\xi}] \hat{\Gamma}_{j}[\boldsymbol{\xi}] \hat{\Gamma}_{J}[\boldsymbol{\xi}]\right], k_{i}(x)=\mathrm{E}\left[\boldsymbol{K}(x) \hat{\Gamma}_{i}(\boldsymbol{\xi})\right]$, as well as the vectors of coordinates $\mathrm{U}_{2}$ and $\theta$ of $\mathbf{U}_{2}$ and $\boldsymbol{\theta}$, respectively. Note that the double indices $(j, \ell)$ and $(J, L)$ each correspond to only one index in the matrix form 
of the system. The mixed system (10) can now be written:

$$
\left[\begin{array}{ccccc}
\mathrm{A}_{1} & 0 & \mathrm{C}_{1} & \kappa_{0} \mathrm{~S}_{1} & 0 \\
0 & \mathrm{~A}_{2} & -\mathrm{C}_{2} & -\kappa_{0} \mathrm{~S}_{2} & 0 \\
\mathrm{C}_{1}^{T} & -\mathrm{C}_{2}^{T} & 0 & 0 & \mathrm{~S}_{c}^{T} \\
\kappa_{0} \mathrm{~S}_{1}^{T} & -\kappa_{0} \mathrm{~S}_{2}^{T} & 0 & 0 & 0 \\
0 & 0 & \mathrm{~S}_{c} & 0 & 0
\end{array}\right]\left[\begin{array}{c}
\mathrm{U}_{1} \\
\mathrm{U}_{2} \\
\Psi \\
\theta \\
\Lambda
\end{array}\right]=\left[\begin{array}{c}
\mathrm{F}_{1} \\
\mathrm{~F}_{2} \\
0 \\
0 \\
0
\end{array}\right]
$$

Although the form of this matrix system is very similar to that of Eq. (32), the size of the latter is much smaller than the former because $\mathrm{A}_{2}$ is a $\mathrm{Nm}_{2} \times \mathrm{Nm}_{2}$ matrix while $\mathbf{A}_{2}$ is a $m_{2} \times m_{2}$ matrix, with $N$ often very large. Note also that, for notational simplicity, we have used the same name $\mathrm{F}_{2}$ for two different quantities in the two systems. The detail of these quantities is irrelevant here.

\subsubsection{Monte-Carlo approach}

We now turn to a discretization of the random dimension based on the Monte-Carlo approach. The general idea is to note that only $\mathbf{A}_{2}, \mathbf{U}_{2}$, and $\boldsymbol{\theta}$ are random in equation (32), and to perform a static condensation of the large matrix with respect to the two latter quantities.

As the part of the stiffness matrix that should be condensated is not necessarily positive definite, we introduce an additional set of degrees of freedom $\boldsymbol{\beta}$ to control the rigid-body modes of the coupling area (see more details in the literature of domain partitioning, for example [21]). We therefore introduce

$$
\mathrm{B}=\left[\begin{array}{cc}
0 & \mathrm{~S}_{1} \\
-\mathrm{C}_{2}^{T} & 0 \\
0 & 0
\end{array}\right]
$$




$$
\mathrm{A}^{*}=\left[\begin{array}{ccc}
\mathrm{A}_{1} & \mathrm{C}_{1} & 0 \\
\mathrm{C}_{1}^{T} & 0 & \mathrm{~S}_{c}^{T} \\
0 & \mathrm{~S}_{c} & 0
\end{array}\right]^{-1}
$$

where the inverse matrix should be understood in the pseudo-inverse sense,

$$
\begin{gathered}
\mathbf{K}^{*}=\left[\begin{array}{cc}
\mathbf{A}_{2} & -\mathrm{S}_{2} \\
-\mathrm{S}_{2}^{T} & 0
\end{array}\right]-\mathrm{B}^{T} \mathrm{~A}^{*} \mathrm{~B}, \\
\mathrm{~F}^{*}=\left[\begin{array}{c}
\mathrm{F}_{2} \\
0
\end{array}\right]-\mathrm{B}^{T} \mathrm{~A}^{*}\left[\begin{array}{c}
\mathrm{F}_{1} \\
0 \\
0
\end{array}\right],
\end{gathered}
$$

and a matrix $R$ of vectors spanning the null space of $A^{*}$ (a vector in acoustics and a matrix in elasticity).

The condensation of system (32) then leads to:

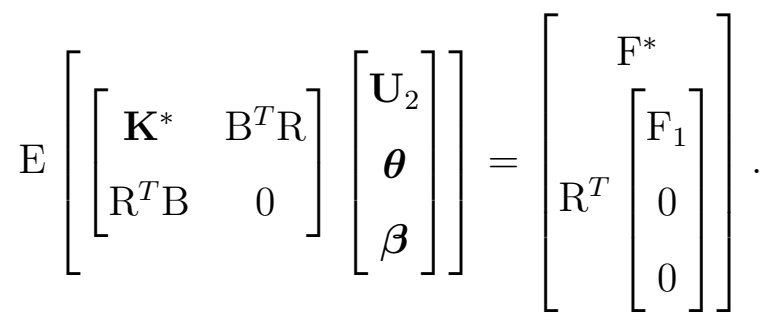

The system (39) can then be solved by the Monte Carlo method. Realizations of $\mathbf{A}_{2}$ are drawn following the appropriate probability law, and statistics of the solution vector $\left[\mathbf{U}^{T} \boldsymbol{\theta}\right]^{T}$ can then be evaluated from the corresponding realizations. The vector of solutions $\left[\mathrm{U}_{1}^{T} \Psi \Lambda\right]^{T}$ is finally retrieved by expansion back to the original system (32).

Remark 3.4. This approach is the one that will be used for the applications proposed in the next section. However, that should not be seen as a statement by the authors that the Monte-Carlo resolution is more efficient than 
the spectral approach. Indeed the matrix system to be solved by Monte Carlo is much smaller, but the matrices are non-sparse. Nevertheless, the implementation of the Monte-Carlo approach is much simpler than the other one, and was chosen here mainly for that reason. Also it should be noted that the rates of convergence of both the Monte Carlo and the spectral approaches are different and dependent and the quantity of interest. Grossly, the Monte Carlo is expected to provide an accurate estimate of the first few moments of a quantity of interest for a reasonable cost, while the spectral solution should be preferred for the estimation of higher moments;

\section{Examples of application}

Two examples are proposed in this section to illustrate the interest of the coupling strategy, in terms of efficiency in particular. For the first one in a mono-dimensional case, we describe the three different possible modeling approaches: deterministic mono-model, stochastic mono-model, and deterministic-stochastic coupled model, and compare the results obtained. For a bi-dimensional case, we assume that the stochastic mono-model is not available, and only the results of the Arlequin method are presented.

\subsection{D Bar in traction}

The first application we describe here is a very simple one that allows a full investigation of the behavior of the method. We consider a simple bar of unit length in traction under imposed displacements, with an unit bulk load. All lengths and stresses are normalized for simplicity. 


\subsubsection{Deterministic mono-model}

The deterministic mono-model of this bar is controlled by a deterministic stiffness $\underline{K}=1$. The displacement field $\underline{u}$ verifies, almost everywhere:

$$
\frac{d}{d x}\left(\underline{K} \frac{d \underline{u}}{d x}\right)+1=0,
$$

with the boundary conditions $\underline{u}(0)=0$ and $\underline{u}(1)=1$. The analytical solution for the deterministic mono-model is $\underline{u}=x(3-x) / 2$ and $d \underline{u} / d x=3 / 2-x$.

\subsubsection{Stochastic mono-model}

The stochastic mono-model is controlled by the stochastic field $\boldsymbol{K}(x)$, which we choose to model as a uniform field with bounds $\ln 2$ and $2 \ln 2$ (arithmetic mean $\mathrm{E}[\boldsymbol{K}]=3 \ln 2 / 2 \approx 1.04$, geometric mean $1 / \mathrm{E}[1 / \boldsymbol{K}]=1$, standard deviation $\sigma_{\boldsymbol{K}}=\ln 2 / 2 \sqrt{3} \approx 0.20$ ), and exponential correlation with correlation length $L_{c}=10^{-2}$. This field obviously verifies conditions (3).

Remark 4.1. A uniform random variable with bounds $0<\alpha<\beta$ has arithmetic mean $(\beta+\alpha) / 2$, geometric mean $\ln (\beta / \alpha) /(\beta-\alpha)$, and standard deviation $(\beta-\alpha) / 2 \sqrt{3}$.

The stochastic displacement field verifies, almost surely and almost everywhere:

$$
\frac{d}{d x}\left(\boldsymbol{K} \frac{d \boldsymbol{u}}{d x}\right)+1=0,
$$

with the boundary conditions $\boldsymbol{u}(0)=0$ and $\boldsymbol{u}(1)=1$, almost surely. The solution for the stochastic mono-model is

$$
\boldsymbol{u}(x)=\int_{0}^{x} \frac{\boldsymbol{K}^{*}-x^{\prime}}{\boldsymbol{K}\left(x^{\prime}\right)} d x^{\prime},
$$


where $\boldsymbol{K}^{*}$ verifies $1=\int_{0}^{1} \frac{\boldsymbol{K}^{*}-x^{\prime}}{\boldsymbol{K}\left(x^{\prime}\right)} d x^{\prime}$ almost surely, and

$$
\frac{d \boldsymbol{u}}{d x}=\frac{\boldsymbol{K}^{*}-x}{\boldsymbol{K}(x)} .
$$

With the parameters above, and assuming that $\boldsymbol{K}^{*} \approx 1 / \mathrm{E}[1 / \boldsymbol{K}]=1$, almost surely, (because the correlation length is much smaller than the total length of the rod, and with some ergodicity assumptions), one gets $\mathrm{E}[\boldsymbol{u}]=x(3-x) / 2$ and $\mathrm{E}[d \boldsymbol{u} / d x]=3 / 2-x$. The standard deviations $\sigma_{\boldsymbol{u}}$ and $\sigma_{d \boldsymbol{u} / d x}$ and other statistical quantities can also be evaluated numerically (using Monte Carlo sampling for example).

In figure 4, we plot the displacements $\underline{u}$ for the deterministic mono-model, as well as the mean $\mathrm{E}[\boldsymbol{u}]$ and the $90 \%$-confidence interval for $\boldsymbol{u}$, for the stochastic mono-model, evaluated using 10000 Monte Carlo trials. In both cases, $\underline{u}$ and $\mathrm{E}[\boldsymbol{u}]$ are perfectly overlapping. This is due to the fact that homogenization is indeed taking place and that $\mathrm{E}[1 / \boldsymbol{K}(x)] \approx 1 / \underline{K}$, almost everywhere.
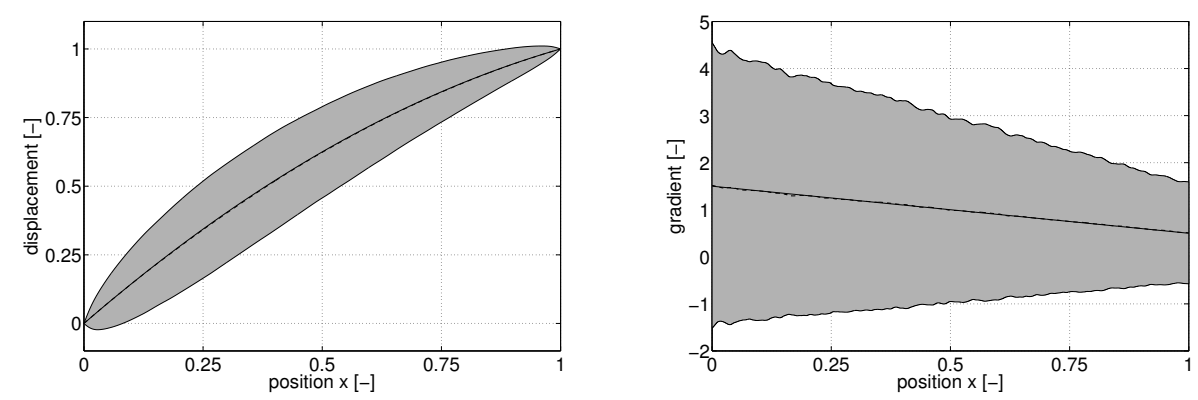

Figure 4: Value of the displacement (left figure) and gradient (right figure) for the deterministic mono-model $\underline{u}$ (solid line), the mean of the stochastic mono-model $\mathrm{E}[\boldsymbol{u}]$ (dashed line, overlapping the solid line) and the 90\%-confidence interval for the stochastic monomodel (grey patch). 


\subsubsection{Deterministic-stochastic coupled approach}

We now move on to the Arlequin coupling model, for which we choose a domain $\Omega_{1}=[0,1]$, a domain $\Omega_{2}=[0.3,0.7]$, and a coupled zone $\Omega_{c}=$ $[0.3,0.5] \cup[0.6,0.7]$. The domain $\Omega_{1}$ is discretized using 10 elements of constant length 0.1 . The domain $\Omega_{2}$ is discretized using 4000 elements of constant length $10^{-4}$. The meshes are therefore embedded and no mesh interpolation is required. In the Arlequin formulation, the mediator space $\mathcal{W}_{c}$ defined by Eq. (15) is chosen here with $\mathcal{V}_{c}=\mathcal{V}_{2}$. The coupled zone is therefore discretized following the same mesh as that of domain $\Omega_{2}$. The Monte-Carlo approach was followed, as described in section 3.4.2, and 10000 samples were used. In this 1D case, the results are obtained almost instantaneously. The results for $u_{1}$ (on domain $\Omega_{1}$ ) and $\boldsymbol{u}_{2}$ (on domain $\Omega_{2}$, in terms of the mean and $90 \%$-confidence interval) are plotted in figure 5, and compared to the results obtained with the stochastic mono-model in figure 6 .
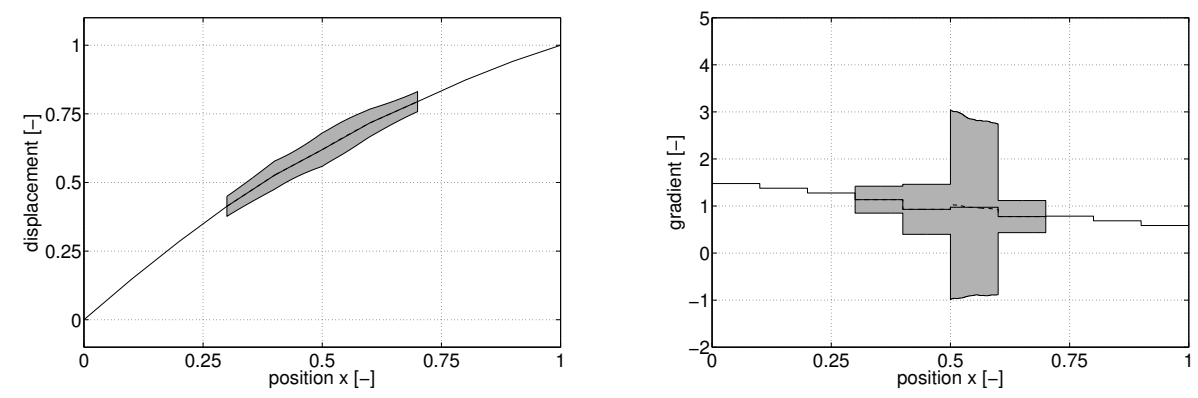

Figure 5: Displacement (left) and gradient of the displacement (right) for the solution of the Arlequin problem in terms of $u_{1}$ (solid line), $\mathrm{E}\left[\boldsymbol{u}_{2}\right]$ (dashed line, overlapping the solid line over $\Omega_{2}$ ), and 90\%-confidence interval for $\boldsymbol{u}_{2}$ (grey patch).

It can be observed that the averages of both the displacement and the gradient, as well as the confidence interval for the gradient compare well on 

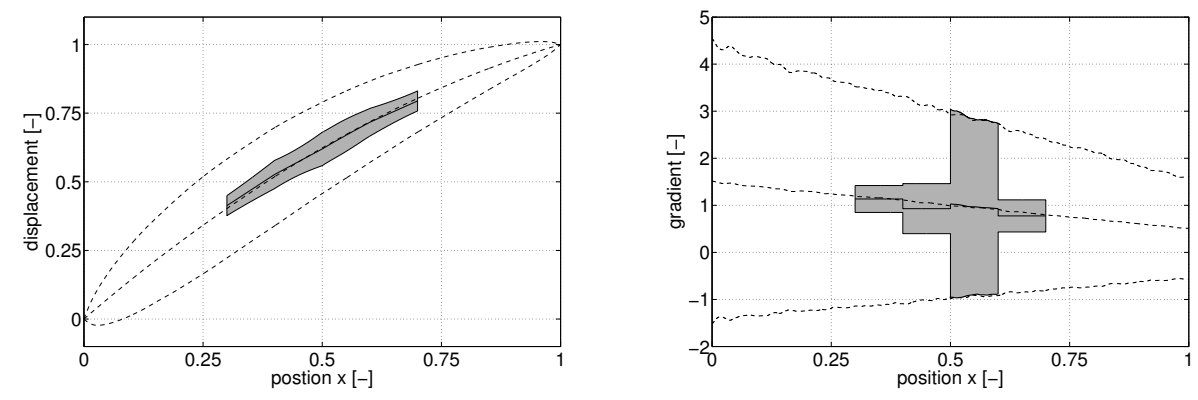

Figure 6: Comparison, in terms of displacement (left) and gradient of the displacement (right), of the Arlequin solution $\boldsymbol{u}_{2}$ (solid line for the mean and grey patch for the $90 \%$ confidence interval) and the solution of the stochastic mono-model $\boldsymbol{u}_{s}$ (dashed lines for the mean and the $90 \%$-confidence interval).

the part where the second model expresses itself (that is $\Omega_{2} \backslash \Omega_{c}=[0.5,0.6]$ ). However this is not the case for confidence interval of the displacement. To analyze this, we plot in figure 7 the correlation between the value of the displacement $\boldsymbol{u}$ at the position $x=0.55$ (which is a random variable) with the values of $\boldsymbol{K}$ at all positions (which is a random field). For this 1D case, the gradient at $x=0.55$ is here a very local quantity (in the sense that it is not influenced by the variability of $\boldsymbol{K}(x)$ at distant positions), while the displacement in $x=0.55$ is not.

In the same figure 7 , we plot the same two correlations but evaluated based on the Monte-Carlo samples used in the Arlequin model, therefore only available over $\Omega_{2}$. Except the faster decrease of the correlation for the displacement, which is due to the influence of the shape function $\alpha_{2}$ in factor of $\boldsymbol{K}$ in the integrals of the weak formulation (see equation (12)), they are perfectly well represented. This seems to indicate that it is possible, only based on the Arlequin solution, to evaluate the quality of the estimation of 

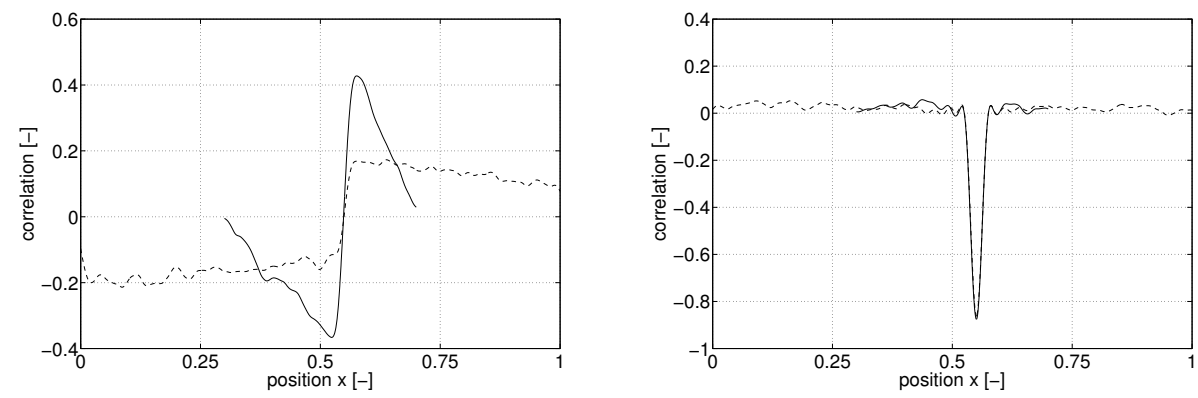

Figure 7: Value of the correlation between the random field $\boldsymbol{K}(x)$ at all points and the displacement in position $x=0.55$ (left figure) and the gradient in position $x=0.55$ (right figure), for the stochastic mono-model (dashed line) and the Arlequin model (solid line).

the confidence interval for a given quantity of interest by considering the localization of the correlation of that quantity of interest with the stochastic data (here $\boldsymbol{K}$ ). More specifically, and before further studies, it could be said that the support of the correlation should be included in the non-coupled zone of the model (that is $\Omega_{2} \backslash \Omega_{c}=[0.5,0.6]$ ). Further research is however needed to refine this idea. It will be discussed in a forthcoming paper.

\subsection{D sample in traction}

We now consider a $2 \mathrm{D}$ sample $\Omega$ inscribed in the box $[-3,3] \times[-1,1]$ (see figure 8). The sample is submitted to an imposed displacement, with no bulk load. The boundary conditions are $\boldsymbol{u}(x=-3, y)=0, \boldsymbol{u}(x=3, y)=1$, and $\nabla \underline{u} \cdot \boldsymbol{n}=0$ for the other edges, almost surely.

The Arlequin model is used to approximate the solution of the previous problem by coupling a deterministic model described by a stiffness $\underline{K}=1$ with a stochastic one described by a stochastic stiffness $\boldsymbol{K}(x)$, modeled as an uniform field with bounds 0.3194 and 2.3027, and exponential correla- 


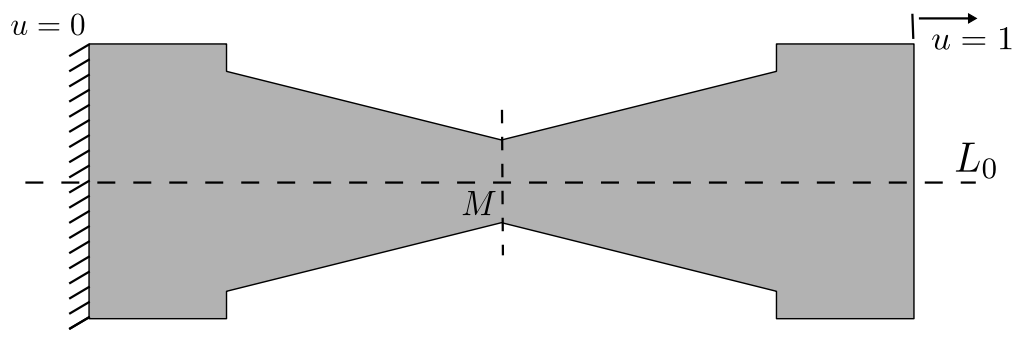

Figure 8: Geometry of the 2D sample

tion with correlation length $L_{c}=0.2$ in each direction. We choose the domain $\Omega_{1}$ to cover the entire sample, while the domain $\Omega_{2}$ is given by $\Omega_{2}=\Omega \cap\{(x, y) \mid x \in[-1.2,1.2]\}$, and a coupled zone $\Omega_{c}=\Omega \cap\{(x, y) \mid x \in$ $[-1.2,-0.6] \cup[0.6,1.2]\}$. The domain $\Omega_{1}$, described by a deterministic model, is discretized using 292 elements. The domain $\Omega_{2}$ is discretized using a finer mesh composed of 6272 elements. The meshes are embedded and no mesh interpolation is required. As in the previous case, the space support of the mediator space $\mathcal{W}_{c}$, defined by Eq. (15) is chosen to overlap the space support $\mathcal{V}_{2}$. The coupled zone is therefore discretized following the same mesh as that of domain $\Omega_{2}$. The Monte-Carlo approach was followed, as described in section 3.4.2, and 10000 samples were used. Note that, even on this simple example, it is not possible to solve the full fine scale problem, because the correlation length of the stochastic field is very small and must be larger than the size of the elements in order that the stochastic realizations be well sampled. However, by reducing the zone in which randomness is considered, the stochastic-deterministic Arlequin coupling method allows to overcome the size limitation. 
The displacement and the gradient are investigated along a line $L_{0}$ (defined by $\{(x, y) \mid y=0\}$, see figure 8 ). The results for $u_{1}$ (on domain $\Omega_{1}$ ) and $\boldsymbol{u}_{2}$ (on domain $\Omega_{2}$, in terms of the mean and 90\%-confidence interval) are plotted in figure 9 .
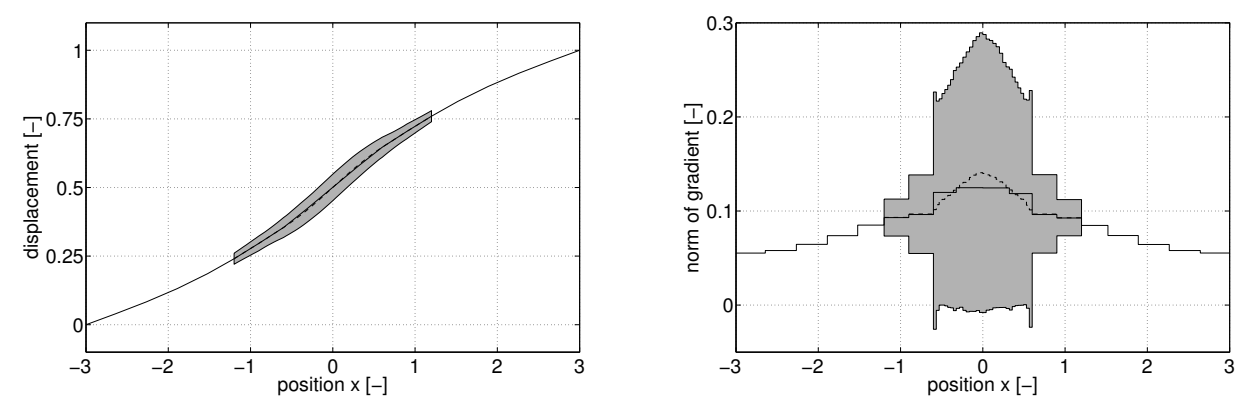

Figure 9: Displacement (left) and gradient of the displacement (right) for the solution of the Arlequin problem in terms of $u_{1}$ (solid line), $\mathrm{E}\left[\boldsymbol{u}_{2}\right]$ (dashed line, overlapping the solid line), and 90\%-confidence interval on $\boldsymbol{u}_{2}$ (grey patch).

As in the previous example, we plot in figure 10 the correlation between the value of the displacement $\boldsymbol{u}$ at the position defined by the point $M$ of coordinates $(x=0, y=0)$ (which is a random variable) with the values of $\boldsymbol{K}(x)$ everywhere on the domain (which is a random field). As previously,
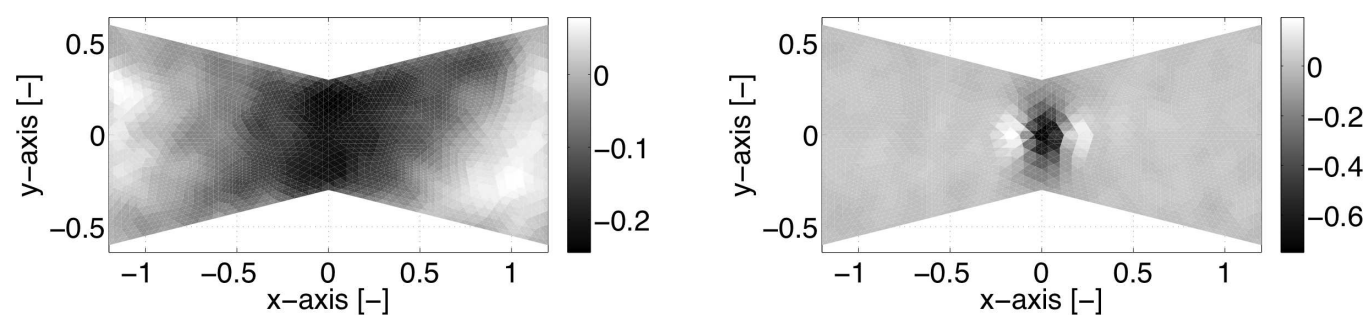

Figure 10: Value of the correlation between the random field $\boldsymbol{K}(x)$ in all elements and the displacement (left figure) and gradient (right figure) at position $M=(x=0, y=0)$ for the Arlequin model. 
we observe that the correlation for the gradient is much more local than that for the displacement. Here, the solution for the stochastic mono-model is not available, so that more precise conclusions cannot be drawn, but we expect the same as before to hold true: the gradient evaluated by the Arlequin method is a good approximation to the gradient evaluated using the monomodel solution, while this is not the case for the displacement. It is expected that the support of the correlation should be included in the free zone of the model (that is $\Omega_{2} \backslash \Omega_{c}$ ). Further research is however needed in that direction.

\section{Conclusions}

In this paper, we have presented a method that allows to couple two models, one stochastic and one deterministic. The presentation, and in particular the choice of the mediator space, was mainly concentrated on the random dimension. The "space" part of the coupling was chosen in a very similar way as in deterministic-deterministic coupling cases considered previously in the Arlequin framework. Hence, it is expected that the pattern followed in this paper can be extended quite straightforwardly to other type of coupling, and in particular to those for which the two models are different (here both models were continuum models).

The formulation that is proposed is both elegant, in the sense that the complexity is localized only where needed, and computationally reasonable, as this decreasing complexity goes along with a lower numerical cost. Both

the existence and uniqueness of the solution of the mixed problem and numerical considerations for the computation of approximations of that solution have been considered. Finally, a first attempt to quantify the appropriate- 
ness of the approach for the evaluation of local quantities of interest has been discussed. This will be completed in a future work by a more general approach for adaptive simulation within the stochastic-deterministic Arlequin framework.

\section{Acknowledgements}

This work was partially supported by the ANR project TYCHE (Advanced methods using stochastic modeling in high dimension for uncertainty modeling, quantification and propagation in computational mechanics of solids and fluids), with project number ANR-2010-BLAN-0904, and by grants from DIGITEO and Région Ile-de-France, with project number 2009-26D.

\section{References}

[1] R. G. Ghanem, R. Kruger, Numerical solution of spectral stochastic finite element systems, Comp. Meths. Appl. Mech. Engr. 129 (1996) 289-303.

[2] M. F. Pellissetti, R. G. Ghanem, Iterative solution of systems of linear equations arising in the context of stochastic finite elements, Adv. Engng. Sftw. 31 (2000) 607-616.

[3] A. Doostan, R. G. Ghanem, J. Red-Horse, Stochastic model reduction for chaos representation, Comp. Meths. Appl. Mech. Engr. 196 (2007) 3951-3966.

[4] A. Sarkar, N. Benabbou, R. G. Ghanem, Domain decomposition of 
stochastic PDEs: theoretical formulations, Int. J. Numer. Meths. Engr. 77 (2009) 689-701.

[5] R. Cottereau, H. Ben Dhia, D. Clouteau, Localized modeling of uncertainty in the Arlequin framework, in: R. Langley, A. Belyaev (Eds.), Vibration Analysis of Structures with Uncertainties, IUTAM Bookseries, Springer, 2010, pp. 477-488.

[6] E. Savin, D. Clouteau, Elastic wave propagation in a 3-D unbounded random heterogeneous medium coupled with a bounded medium. Application to seismic soil-structure interaction (SSSI), Int. J. Numer. Meths. Engr. 54 (2002) 607-630.

[7] L. Chamoin, J. T. Oden, S. Prudhomme, A stochastic coupling method for atomic-to-continuum Monte-Carlo simulations, Comp. Meths. Appl. Mech. Engr. 197 (2008) 3530-3546.

[8] A. Ern, J.-L. Guermond, Theory and practice of finite elements, volume 159 of Applied Mathematical Sciences, Springer, 2004.

[9] I. Babuška, R. Tempone, G. E. Zouraris, Galerkin finite element aproximations of stochastic elliptic partial differential equations, SIAM J. Numer. Anal. 42 (2004) 800-825.

[10] R. G. Ghanem, P. D. Spanos, Stochastic finite elements: a spectral approach, Springer-Verlag, 1991.

[11] G. Stefanou, The stochastic finite element method: past, present and future, Comp. Meths. Appl. Mech. Engr. 198 (2009) 1031-1051. 
[12] C. P. Robert, G. Casella, Monte Carlo statistical methods, Springer, 2004 .

[13] H. Ben Dhia, G. Rateau, The Arlequin method as a flexible engineering design tool, Int. J. Numer. Meths. Engr. 62 (2005) 1442-1462.

[14] H. Ben Dhia, Multiscale mechanical problems: the Arlequin method, Comptes Rendus de l'Académie des Sciences - Series IIB 326 (1998) 899-904.

[15] H. Ben Dhia, G. Rateau, Mathematical analysis of the mixed Arlequin method, Comptes Rendus Acad. Sci. - Series I - Math. 332 (2001) 649654 .

[16] H. Ben Dhia, Further insights by theoretical investigations of the multiscale Arlequin method, Int. J. Multiscale Comp. Engr. 6 (2008) 215-232.

[17] S. P. Xiao, T. Belytschko, A bridging domain method for coupling continua with molecular dynamics, Comp. Meths. Appl. Mech. Engr. 193 (2004) 1645-1669.

[18] G. W. Milton, The theory of composites, Cambridge Monographs on Applied and Computational Mechanics, Cambridge University Press, 2002.

[19] F. Brezzi, On the existence, uniqueness and approximation of saddle-point problems arising from lagrangian multipliers, Revue Française d'automatique, informatique, recherche opérationnelle. Analyse numérique 8 (1974) 129-151. 
[20] C. Soize, R. G. Ghanem, Physical systems with random uncertainties: chaos representations with arbitrary probability measures, SIAM J. Sci. Comp. 26 (2004) 395-410.

[21] C. Farhat, F.-X. Roux, A method of finite element tearing and interconnecting and its parallel solution algorithm, Int. J. Numer. Meths. Engr. 32 (1991) 1205-1227. 